\title{
Comparison of Decentralized ADMM Optimization Algorithms for Power Allocation in Modular Fuel Cell Vehicles
}

\author{
Arash Khalatbarisoltani, Member, IEEE, Mohsen Kandidayeni, Member, IEEE, Loïc Boulon, Senior \\ Member, IEEE, Xiaosong Hu, Senior Member, IEEE
}

\begin{abstract}
The advanced modular powertrains are envisioned as primary part of future hybrid fuel cell vehicles (FCVs). The existing papers in the literature solely cope with the hardware side of modularity, while the software side is also vital to capitalize on the total capacity of these powertrains. Driven by this motivation, this paper puts forward a comparative study of two novel decentralized convex optimization frameworks based on alternating direction method of multipliers (ADMM) to solve a multi-objective power allocation strategy (PAS) problem in a modular FCV (MFCV). The MFCV in this work is composed of two fuel cell (FC) stacks and a battery pack. Despite the existing centralized strategies for such a modular system, this manuscript proposes two decentralized PASs (Dec-PASs) based on Consensus ADMM (C-ADMM) and Proximal Jacobian ADMM (PJ-ADMM) to bridge the gap regarding the appreciation of modularity in software terms. Herein, after formulating the central PAS optimization problem, the principle of utilizing such decentralized algorithms is presented in detail. Subsequently, the performance of the proposed Dec-PASs is examined through several numerical simulations as well as experiments on a developed small-scale test bench. The obtained results illustrate that decomposition into decentralized forms enables solving the complex PAS optimization problem faster and provides modularity and flexibility. Furthermore, the proposed Dec-PASs can cope with fault and malfunction and thus augment the durability and robustness of modular powertrain systems.
\end{abstract}

Index Terms - Alternating direction method of multipliers (ADMM), distributed optimization, energy management, fuel cell hybrid vehicle, proton exchange membrane fuel cell (PEMFC).

\section{INTRODUCTION}

\section{A. General context}

$\mathrm{O}$ ne of the largest sources of greenhouse gas emissions is burning fossil fuels for the transportation sector. In this regard, electrification of transportation through different hybrid and pure electric vehicle technologies has come under attention [1]. Fuel cell vehicles (FCVs), which are generally composed of a fuel cell system (FCS) as the primary power source and an energy storage system (battery and/or supercapacitor) as the secondary one, are considered as one of the promising solutions

This work was supported in part by the Natural Sciences and Engineering Research Council of Canada (NSERC) and the Canada Research Chairs program. The work of $\mathrm{X}$. Hu was supported by the Chongqing Natural Science Foundation for Distinguished Young Scholars (Grant No. cstc2019jcyjjq0010), Chongqing Science and Technology Bureau, China. (Corresponding authors: Loïc Boulon and Xiaosong Hu.) A. Khalatbarisoltani, and L. Boulon are with the Hydrogen Research Institute, Department of Electrical and Computer Engineering, Université du Québec à Trois-Rivières, QC G8Z 4M3, Canada (arash.khalatbarisoltani@uqtr.ca, loic.boulon@uqtr.ca). M. Kandidayeni is with Electric-Transport, Energy Storage and Conversion Lab (e-TESC) at Université de Sherbrooke and a research assistant in UQTR Hydrogen Research Institute. (mohsen.kandidayeni@usherbrooke.ca) X. Hu is with the State Key Laboratory for Mechanical Transmission, Department of Automotive Engineering, Chongqing University, Chongqing 400044, China, and the Advanced Vehicle Engineering Centre, Cranfield University, Cranfield, MK43 0AL, UK (xiaosonghu@ieee.org). to mitigate this critical concern $[2,3]$. Among different FCSs, proton exchange membrane (PEM) fuel cell (FC) has been the most appropriate candidate for this application due to its efficiency, power density, low noise, and low-temperature operation range [4]. The lithium-ion battery is also the dominant battery technology in this domain due to its high energy and power density and low self-discharge rate [5]. Since the power sources of an FCV have different energetic characteristics, the use of a power allocation strategy (PAS) is crucial to minimize the total cost (hydrogen consumption and degradation of the components) [6,7]. A large number of studies have been done on the design of PASs for FCVs, such as rule-based [8,9], fuzzy-based [10-14], optimization-based [15-19], predictive-based [20-23], and adaptive strategies [24]. The majority of these studies are based on centralized PASs (Cen-PAS) designed for a single-stack FCV. Hence, they are vulnerable to the malfunction of the power sources, which can occur in such a configuration. This weakness exacerbates in high-power applications, like buses and trucks, since many cells must be stacked to meet the requested power. Consequently, with all the advantageous of the single-stack FCSs, it is necessary to advance them in terms of durability, modularity, and efficiency.

\section{B. Literature study}

One of the proposed promising solutions in this regard is the use of modular FCSs (MFCSs) [25]. Unlike the typical FCVs, the modular FCVs consist of several connected low-power FCs rather than a high-power one along with an energy storage system. In [26], a survey of the MFCSs with different power conditioning topologies and fluidic architectures is provided and concluded that these systems offer better efficiency and lower hydrogen consumption than the single-stack ones. Moreover, the inherent redundancy of the MFCSs leads to the increase of robustness and reliability in case of malfunction occurrence in one of the FCs and/or the converters. On the other hand, the extra degrees of freedom in the MFCSs necessitate the design of advanced PASs to optimize the end-user costs and fulfill the powertrain system requirements.

There are several research efforts based on the Cen-PASs for MFCVs. For instance, in [27], three PASs, namely daisy chain, equal distribution, and optimal splitting, are compared for an MFCV, and it is shown that the optimal splitting achieves the best performance. In [28], a rule-based PAS is utilized for an MFCV and concluded that this strategy is suitable for high hybridization ratios. In [29], four FCSs have been connected via 
power converters to the $\mathrm{DC}$ bus using a maximum power point tracking controller for each stack. In [30], a hysteresis PAS is developed for an MFCV composed of three FCSs and a battery pack. The primary purpose of the suggested PAS is to make the operation time of the three FCSs equal while reducing the number of on-off cycles. In [31], an adaptive state machine PAS is proposed to distribute the power among four FCSs and a battery pack. Simultaneously, the FCSs are constantly monitored in terms of their maximum power and efficiency points. In [32], an adaptive current distribution method based on a droop control technique is proposed for two FCSs to decline the degradation rate.

Although the discussed modular powertrains bring about modularity and reliability from electrical and fluidic (hardware) points of view, they do not guarantee these aspects in their management and control units (software). These Cen-PASs are susceptible to a precipitously single point of failure through their software programs. Moreover, the additional degrees of freedom make these Cen-PASs substantially complex and timeconsuming to be solved in real-time, which is a critical aspect in the FCV applications. Therefore, there has been a growing trend in the literature to gradually shift from Cen-PASs to decentralized PASs (Dec-PASs). For instance, in [33, 34], two Dec-PASs based on game theory are proposed. However, the main drawback of these strategies is that the players are selfish and may not converge to their optimal results. Furthermore, these Dec-PASs cannot entirely satisfy the nonlinearities in the behaviour and the constraints of different sources. Another worth noting problem with these strategies is that they need a lot of data exchange which is not feasible for the onboard applications. In [35], a droop-based Dec-PAS is proposed for seeking optimal power-sharing. However, this approach cannot perform well in a wide range of operations and does not consider the longevity of the powertrain system.

To evade the above-mentioned problems in other domains with multi-source systems, such as smart grids [36, 37], special attention has been given to decentralized convex optimization (DCO) algorithms [38]. One of the most famous classical decomposition methods in this regard is the one introduced in [39] based on Lagrangian Relaxation. However, this method has slow convergence. Several other methods, such as auxiliary problem principle (APP) [40], consensus-based algorithm [41], Karush-Kuhn-Tucker (KKT) conditions [42], and alternating direction method of multipliers (ADMM) [43, 44], have been proposed to enhance the convergence rate. Among them, ADMM has attracted a lot of attentions since it can guarantee the global convergence and does not require a significant amount of data exchange in spite of other algorithms. This method amalgamates dual decomposition with the multipliers technique and the augmented Lagrangian approach. ADMM decomposition-based method can be categorized into GaussSeidel ADMM (GS-ADMM), Variable Splitting ADMM (VSADMM), and Jacobian ADMM (J-ADMM) [45]. GS-ADMM cannot be straightforwardly applied to an optimization problem with more than three subproblems and hence cannot guarantee the convergence in this case [45]. VS-ADMM is also not practical for large-size optimization problems, and J-ADMM may diverge for various problems although its updating procedure is parallel. In this regard, J-ADMM and GS-ADMM have been advanced to Proximal Jacobian ADMM (PJ-ADMM) and Consensus ADMM (C-ADMM), respectively, to be more practical for the distributed optimization problems. The update processes of PJ-ADMM and C-ADMM are parallel, and convergence performance can be guaranteed simultaneously [46]. These two DCO-based algorithms offer several advantages compared to centralized ones. Firstly, parallel execution feature enables them to solve complex optimization problems with less computational effort. Secondly, they can autonomously adapt to new changes which provides robustness in case of any subsystem failure.

In $[47,48]$, two classic ADMM algorithms are suggested for solving Cen-PASs in hybrid electric vehicles. However, their central control units do not offer modularity, plug \& play aspect, and robustness in terms of software. In [49], an APPbased scheme is proposed for a modular FCV. However, this decentralized approach will not provide satisfying results in convergence speed compared to other advanced DCO algorithms, such as PJ-ADMM and C-ADMM.

\section{Contribution}

In the light of the discussed papers, it can be stated that there is a lack of discussion on designing Dec-PAS via DCOs in the domain of MFCVs. To the best of the authors' knowledge, this work is one of the leading attempts, if any, to propose a practical framework for designing Dec-PASs in MFCVs. In this respect, a detailed comparison of two Dec-PASs based on C-ADMM and PJ-ADMM is put forward for an MFCV. These two decomposition-based approaches are singled out due to their parallel updating process, fast convergence speed, ability to handle constraints, and global convergence performance. The proposed Dec-PASs are compared with an offline optimal PAS (dynamic programming (DP)) and a Cen-PAS (sequential quadratic programming (SQP)). The performance of these fully Dec-PASs is thoroughly explored in terms of final cost under a real driving profile. Since the ADMM-based decentralized approaches are highly sensitive to their parameters tuning, this vital characteristic is thoroughly investigated. Moreover, to further highlight the DCO-based PAS capabilities, the best DecPAS is selected for price sensitivity and dynamic fault robustness analyses. It is worth reminding that the performance of the PJ-ADMM algorithm has been evaluated using a developed experimental test bench, as opposed to most of similar manuscripts in the literature, which are solely based on simulation.

\section{Organization}

The rest of this paper is structured as follows. In section II, the configuration and modeling of the MFCV are described in detail. Section III presents the general formulation of the multiobjective PAS optimization problem. Section IV describes the two utilized decentralized ADMM-based PAS frameworks. In section $\mathrm{V}$, a detailed comparison of several cases is conducted to scrutinize the performance of the proposed DCO-based PASs. Section VI demonstrates the implementation results via a developed small-scale test bench to validate the theoretical background. Section VII recaps the main points of the paper and 
proposes some perspectives.

\section{MFCV POWERTRAIN CONFIGURATION AND MODELING}

\section{A. Powertrain structure and modeling}

To investigate the performance of the proposed Dec-PASs, a modular FC test bench based on an electric vehicle is established [50], as shown in Fig. 1. The developed small-scale test bench comprises two FC modules, a battery pack, a programmable DC electronic load, and a multi-range programmable DC power supply for simulating the requested load profile. The critical components of each FC module are a 500-W open-cathode PEMFCS (Horizon, model: H-500), a smoothing inductor, and an adjustable unidirectional boost DCDC converter (Zahn Electronics ${ }^{\mathrm{TM}}$, model: DC5036-SU). Moreover, six series $12 \mathrm{~V} / 18 \mathrm{Ah}$ battery packs provide the voltage of the DC-bus. Each module has its autonomous DecPAS inside of a National Instrument CompactRIO (NI9022). The optimal reference of each module is calculated at each control instant with an interval of $10 \mathrm{~Hz}$.

a)
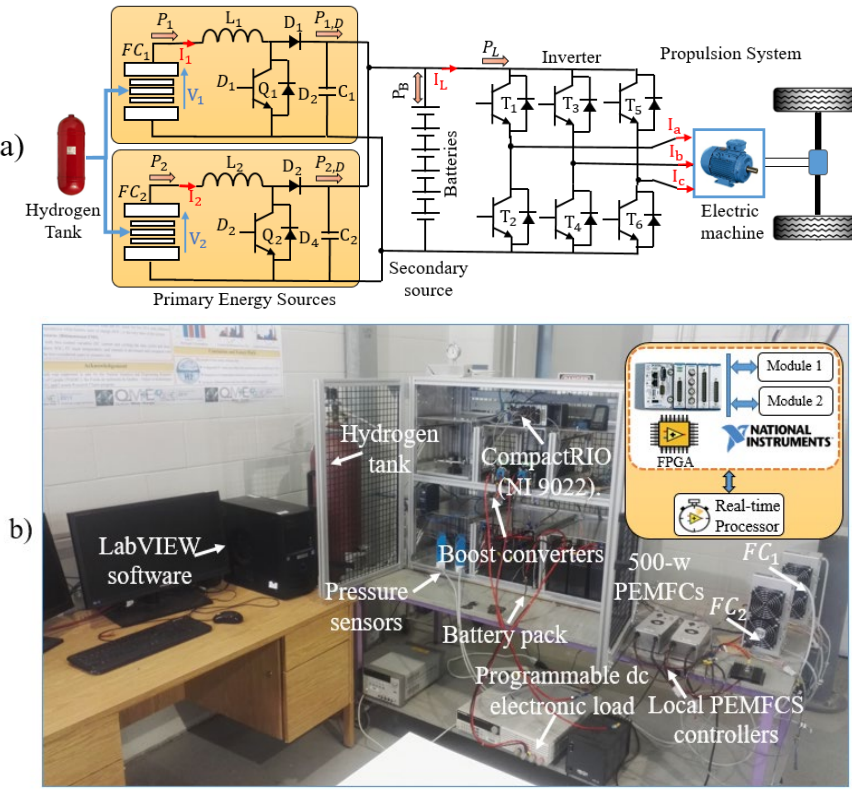

Fig. 1. The developed small-scale test bench: (a) the schematic of the powertrain system, and (b) the developed test bench.

The power equilibrium of the FC modules and the battery unit is formulated in (1).

$$
\sum_{m=1}^{M} P_{m, k} \cdot D_{m, k}+P_{B, k}=P_{L, k},
$$

where $P_{\mathrm{m}}, m \in M, M=\{1,2\}$ denotes the generated power by each one of the $500-\mathrm{W}$ FCSs, $D_{\mathrm{m}}$ is the control signal of the boost converters, $P_{B}$ is the power provided by the battery unit, $P_{L}$ is the requested power from the propulsion system, and $k$ is the time instant.

\section{B. FCS modeling and constraints}

In this work, each of the $500-\mathrm{W} \mathrm{FCSs}, F C_{m}$, are modeled as a voltage source where their polarization curves and the hydrogen mass flows versus requested current are described by experimentally validated quasi-static curves, as shown in Fig. 2. As explained in [14], the output power of an H-500 Horizon FCS is obtained by subtracting the power of the FC stack from the consumed power by the cooling fans and the hydrogen valve. The consumed power by the purge valve is ignored as it has a fixed cyclic purging (every ten seconds for a duration of $100 \mathrm{~ms}$ ). In this paper, the blowers and ancillaries of the FCSs are not explicitly modeled. Nevertheless, their energetic performances are taken into consideration by the static characteristics of the FCSs. In other words, the presented characteristics in Fig. 2 belong to the FCSs considering the fans and hydrogen valve. The technical data of the utilized FCSs are reported in Table I. Fig.3 demonstrates the measured and normalized hydrogen consumptions of the FC modules.
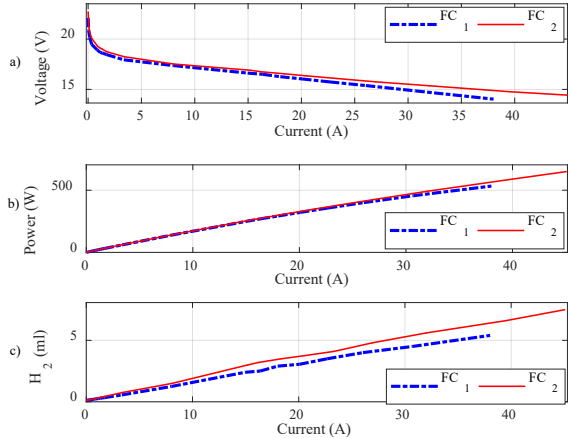

Fig. 2. The characteristic curves of the two real FC modules: (a) polarization curves, (b) power curves, and (c) hydrogen curves.

Table I

Technical specification of the open-cathode PEMFC stacks (H-500)

Number of cells $=24 \quad$ Hydrogen pressure $=0.45-0.55$ bar

Rated power $=500 \mathrm{~W} \quad$ External temperature $=5-30{ }^{\circ} \mathrm{C}$

Rated performance $=14.4 \mathrm{~V} @ 35 \mathrm{~A} \quad$ Max stack temperature $=65^{\circ} \mathrm{C}$

Output voltage range $=12 \mathrm{~V}-24 \mathrm{~V} \quad$ Humidification= Self-humidified

Reactants $=$ Hydrogen and Air $\quad$ Cooling $=$ Air (cooling fan)

Flow rate at $\max$ power $=6.5 \mathrm{~L} / \mathrm{min}$ Efficiency of stack=40\%@14.4V

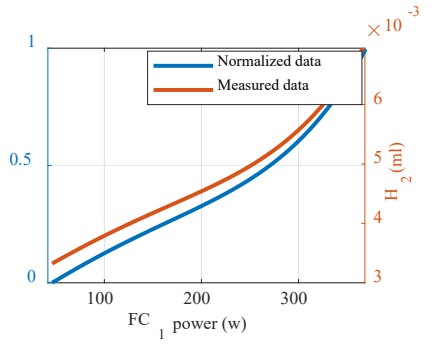

(a)

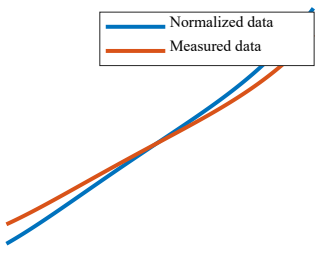

(b)
Fig. 3. The measured and normalized hydrogen consumption curves of the developed test bench: (a) $F C_{1}$ and (b) $F C_{2}$.

Equation (2) enforces power and slew rate limits.

$$
\begin{gathered}
P_{m, \min } \leq P_{m, k} \leq P_{m, \max }, \\
R_{d, m} \Delta t \leq P_{m, k}-P_{m,(k-1)} \leq R_{u, m} \Delta t,
\end{gathered}
$$

where $P_{m, \min }$ and $P_{m, \max }$ are the minimum and maximum values for $P_{m}, R_{d, m}$ and $R_{u, m}$ are boundaries of the slew rates, and $\Delta t$ indicates the time step. As explained in [14], for rising, a dynamic limitation of $10 \%$ of the maximum power per second, and for falling, $30 \%$ of the maximum power per second have been considered for the operation of the FC stack. These constraints prevent the FC stack from sudden changes, which can result in degradation.

\section{Battery modeling and constraints}

A first-order RC model of the battery pack is formulated by $I_{B, k}=\frac{V_{0, k}-R_{S} I_{B, k}-V_{B, k}}{R_{C}}+C_{C} \frac{d}{d t}\left(V_{0, k}-R_{S} I_{B, k}-V_{B, k}\right)$, 
where $I_{B}$ is the battery pack current, $V_{0}$ is the open-circuit voltage, $R_{S}$ is the series ohmic resistance, $V_{B}$ is the output terminal voltage, $R_{c}$ denotes the polarization resistance, and $C_{c}$ is the polarization capacitor. Equation (4) imposes power and slew rate limits.

$$
\begin{gathered}
P_{B, \min } \leq P_{B, k} \leq P_{B, \max }, \\
R_{d, B} \Delta t \leq P_{B, k}-P_{B,(k-1)} \leq R_{u, B} \Delta t,
\end{gathered}
$$

where $P_{B, \min }$ and $P_{B, \max }$ are the minimum and maximum limits of $P_{B}$, respectively, and $R_{d, B}$ and $R_{u, B}$ are the slew rate boundaries of $P_{B}$. Equation (5) presents the state of charge $(S O C)$ calculation formula along with the constraints on the battery $S o C$ level.

$$
\begin{gathered}
S o C_{k+1}=S o C_{k}-\frac{P_{B, k} \Delta t}{Q_{B} V_{B, k} 3600}, \\
S o C_{\text {min }} \leq S o C_{k} \leq S o C_{\text {max }},
\end{gathered}
$$

where $S o C_{\min }$ and $S o C_{\max }$ denote the minimum and maximum limits of $S o C$, respectively, $S o C_{k=0}$ is the initial $S o C$ level, and $Q_{B}$ represents the battery capacity. The battery lifetime is affected by the depth of discharge $(D o D)$ and is defined as an initial capacity drop (reaching $80 \%$ of the initial capacity). The state of health $(\mathrm{SoH})$ is calculated by

$$
\begin{gathered}
\mathrm{SoH}_{k+1}=\mathrm{SoH}_{k}-\frac{\left|P_{B, k}\right| \Delta t}{2 n_{B} Q_{B} V_{B, k} 3600}, \\
\mathrm{SoH}_{\text {min }} \leq \mathrm{SoH}_{k},
\end{gathered}
$$

where $\mathrm{SoH}_{\min }$ and $\mathrm{SoH}_{k=0}$ indicate the minimum and initial $\mathrm{SoH}$, respectively, and $n_{B}$ is the total number of cycles during the whole lifetime of the battery pack. The parameters of the battery pack have been obtained from experimental tests $\left(V_{0}=\right.$ $12.21 \mathrm{~V} \quad, \quad R_{s}=0.0141 \Omega \quad, \quad Q_{B}=18.2 \mathrm{Ah}$ , and $R_{c}=0.0177 \Omega$ ).

\section{Boost converter modeling and characteristics}

The two converters are modeled as follows.

$$
\begin{gathered}
L_{m} \frac{d}{d t} I_{m, k}=V_{m, k}-V_{h, m, k}-r_{m} I_{m, k}, \\
\left\{\begin{array}{c}
V_{h, m, k}=m_{h, m} V_{B, k} \\
I_{h, m, k}=m_{h, m} I_{m, k} \eta_{m}^{z}
\end{array}=\left\{\begin{array}{c}
1, \text { if } P_{m}>0 \\
-1, \text { if } P_{m}<0
\end{array}\right.\right.
\end{gathered}
$$

where $I_{m}$ and $V_{m}$ are the current and voltage of $F C_{m}$, respectively, $L_{m}$ presents the smoothing inductor inductance, $r_{m}$ is the smoothing inductor resistance, $\eta_{h, m}$ is the average efficiency, and $m_{h, m}$ is the modulation ratio of the converters. The estimated parameters of the boost converters are $L_{m}=$ $1.1 \mathrm{mH}, r_{m}=23.9 \mathrm{~m} \Omega$, and $\eta_{m}=96.21 \%$.

\section{Process of DeVeloping the General PAS PRoblem AND THE EVALUATION FUNCTION}

This section firstly presents a standard framework for formulating the main PAS optimization problem (8). Next, an evaluation function (9) is defined to measure all the main criteria used in different optimization methods in the same way.

\section{A. Formulation of the central PAS Problem}

The multi-objective optimization problem of the MFCV can be written as follows.

$$
\min \sum_{m=1}^{M} g\left(c_{m, k}+c_{B, k}\right),
$$

$P_{1}, \ldots, P_{M}, P_{B}$

s.t $\sum_{m=1}^{M} A_{m} P_{m}=c$, other equality, and inequality constraints where $g$ is a convex approximation cost function to be minimized, including the FC modules' normalized hydrogen consumption, and the normalized degradation of the modules, $\mathrm{c}_{m, k}$, and the battery system, $\mathrm{c}_{B, k}$, which are formulated as follows.

$$
c_{m, k}=\mathrm{s}_{h, m, k}+\mathrm{s}_{d, m, k}, c_{B, k}=s_{B, k}+s_{S o C, k}
$$

where $P_{m} \in \mathbb{R}^{N_{m}}$ stands for the control actions, $A_{m} \in \mathbb{R}^{M \times N_{m}}$ and $c \in \mathbb{R}^{M}$ apply the powertrain and the coupling constraints to the modules, respectively, $k$ stands for each simulation moment, and $\mathrm{s}_{h, m, k}$ is the normalized hydrogen consumption function, calculated by

$$
\mathrm{s}_{h, m, k}=\frac{h_{m, k}-h_{m, \min }}{h_{m, \max }-h_{m, \min }},
$$

where $h_{m, k}, h_{m, \min }$, and $h_{m, \max }$ are the hydrogen consumption, and the minimum and maximum limits of $F C_{m}$, respectively. The normalized $F C_{m}$ degradation term, $\mathrm{s}_{d, m}$, which includes two normalized cost shaping functions, the low-power degradation, $s_{d, m}^{l}$, and the high-power degradation, $s_{d, m}^{h}$, are formulated by

$$
s_{d, m, k}=\alpha_{l} s_{d, m, k}^{l}+\alpha_{h} s_{d, m, k}^{h},
$$

where $s_{d, m}^{l}$ and $s_{d, m}^{h}$ are computed by

$$
\begin{aligned}
& s_{d, m, k}^{l}=1-\frac{\left(P_{\operatorname{m}, k}-P_{\operatorname{m}, \min }\right)^{2}}{\left(P_{\max }-P_{\operatorname{man}}\right)^{2},} \\
& s_{d, m, k}^{h}=1-\frac{\left(P_{m, k}-P_{\max }\right)^{2}}{\left(P_{m, \max }-P_{\min }\right)^{2},}
\end{aligned}
$$

where $\alpha_{l}$ and $\alpha_{h}$ are the constant coefficients, computed by

$$
\alpha_{l}=\frac{\varepsilon_{l}}{\varepsilon_{l}+\varepsilon_{h}}, \alpha_{h}=\frac{\varepsilon_{h}}{\varepsilon_{l}+\varepsilon_{h}},
$$

where $\varepsilon_{l}$ and $\varepsilon_{h}$ are the low-power and high-power cell degradation rates, respectively. The degradation terms are adopted from the previous studies since several long-duration aging tests, which are beyond the scope of this research work, are needed to determine them. These values are modified based on the number of cells in the utilized FCSs. The values of these variables $\left(\varepsilon_{l}=8.662 \mu \mathrm{V} / \mathrm{h}\right.$ and $\left.\varepsilon_{h}=10 \mu \mathrm{V} / \mathrm{h}\right)$ have been obtained from $[51,52]$. The normalized battery pack degradation function, $s_{B}$, is calculated by

$$
S_{B, k}=\frac{P_{B, k}}{P_{L, \max }},
$$

where $P_{L, \text { max }}$ denotes the maximum value of $P_{L} \cdot S_{S o C}$ is a punishment term to measure the $\mathrm{SoC}$ level variation, which is defined by

$$
s_{S o C, k}=\beta\left(S_{0} C_{k}-S o C_{k=0}\right)^{2},
$$

where $\operatorname{SoC}_{k=0}$ is the initial $\operatorname{SoC}$, and $\beta$ is a big positive coefficient.

\section{$B$. The defined evaluation function}

The end-user cost, $S_{T}$, in USD, is calculated by

$$
S_{T}=\left(\sum_{K} \sum_{m} S_{H, m, k}+S_{d, m, k}+S_{B, k}\right)+S_{S o C} \text {, }
$$

The hydrogen cost of each module, $S_{H, m}$, is computed by

$$
S_{H, m, k}=h_{m, k} C_{H_{2}} \Delta t \text {, }
$$

where $h_{m, k}$ is the hydrogen consumption, $C_{H_{2}}$ is the hydrogen price, and $\Delta t$ indicates the time step. The degradation cost of each module, $S_{d, m, k}$, is calculated by

$$
\mathrm{S}_{d, m, k}=S_{d, m, k}^{l}+S_{d, m, k}^{h},
$$

where $S_{d, m, k}^{l}$ and $S_{d, m, k}^{h}$ are costs of low-power and high-power degradation, given by

$$
S_{d, m, k}^{l}=\frac{n_{m} \varepsilon_{l} C_{F C, m} \Delta t \mu_{l, m}}{3600 V_{n, m}}, S_{d, m, k}^{h}=\frac{n_{m} \varepsilon_{h} C_{F C, m} \Delta t \mu_{h, m}}{3600 V_{n, m}},
$$


where $n_{m}$ represents the numbers of cells in each $F C_{m}, V_{n, m}$ is $10 \%$ of the nominal $F C_{m}$ voltage drop, and $C_{F C_{m}}$ is FCS cost. $\mu_{l, m}$ and $\mu_{h, m}$ are equal to

$$
\begin{aligned}
& \mu_{l, m}=\left\{\begin{array}{l}
1, \text { if } P_{\min , m} \leq P_{m, k} \leq 0.2 P_{n o m, m} \\
0, \quad \text { otherwise. }
\end{array},\right. \\
& \mu_{h, m}=\left\{\begin{array}{ll}
1, & \text { if } 0.8 P_{n o m, m} \leq P_{m, k} \leq P_{\max , m} \\
0, & \text { otherwise. }
\end{array},\right.
\end{aligned}
$$

where $P_{\text {nom, } m}$ is the output power recommended by the manufacturing company for nominal use of the FCS [52]. The battery degradation cost, $S_{B}$, is determined by

$$
S_{B, k}=C_{B}\left(\mathrm{SoH}_{B, k}-\mathrm{SoH}_{B, 0}\right) \text {, }
$$

where $C_{B}$ is the battery price. The punishment term, $S_{S o C}$, is to recharge the battery to reach the initial $S o C$ by $F C_{m}$ while operating at their maximum efficiency points. The reference prices are $C_{H_{2}}=3.9254 \$ / \mathrm{Kg}$ [53], battery $C_{B}=189 \$ / \mathrm{kWh}$ [54], and the FCS $C_{F C_{m}}=35 \$ / \mathrm{kW}[55]$.

\section{FORMULATION OF THE GENERAL ADMM PAS FRAMEWORKS}

In this section, the utilized DCO algorithms are described. To apply the DCO-based algorithms, firstly, the augmented Lagrangian functions of the power-split problem are derived. Subsequently, the corresponding functions are decomposed, and the broken-down terms are minimized. Finally, the dual variables are updated. These procedures continue until the convergence criteria are satisfied.

It is worth noting that for the following two subsections, each variable with the index of $j$ means the current value at the $j^{t h}$ iteration, and each variable with the index of $j+1$ denotes the new value. $k$ is the position in the selected driving profile. $P_{1, k}$ and $P_{1 . k}^{S_{2}}$ are related to $F C_{1}$, and $P_{2, k}^{S_{1}}$ and $P_{2, k}$ are linked to $F C_{2}$. During the optimization process, each decentralized controller calculates the power of $F C_{1}$ and $F C_{2}$ at the same time. For instance, the decentralized controller 1 solves its subproblem for one iteration and calculates the output power $P_{1, k}$ for its module and $P_{2, k}^{S_{1}}$ for the neighboring module. The decentralized controller 2 solves its subproblem for one iteration and calculates the output power $P_{2, k}$ for its module and $P_{1, k}^{S_{2}}$ for the neighboring module. During the exchange step, $P_{1, k}^{S_{2}}$ is sent to $F C_{1}$ and $P_{2, k}^{s_{1}}$ is sent to $F C_{2}$. Then, these values will be used in the next iteration of the optimization procedure. These shared variables are used to ensure that all the constraints are fulfilled for the final results in the $k^{\text {th }}$ step. The final power of $P_{1, k}$ and $P_{2, k}$ will be sent to the converters as $P_{1, k}^{r e f}$ and $P_{2, k}^{r e f}$ to control the modules and the rest of the requested power will be supplied by $P_{B}$. These final values will be used for the $k^{t h}$ step. For each $k$, during the optimization iterations, the state variables of the modular powertrain system are supposed to be unchanged. However, these values will be updated in the next step $(k+1)$.

\section{A. C-ADMM-based PAS}

As shown in Fig. 4, the central PAS is decomposed into two coupled subproblems, $s_{m}$. Since the coupling constraints are not separable, $P_{m, k}$ is copied into its neighboring module and linked with a global power vector, $w_{G, k}=\left\{w_{1, k}, w_{2, k}\right\}$. In this respect, $P_{1, k}$ is copied into $s_{2}, P_{1, k}^{s_{2}}$, as a virtual power, and $w_{1, k}$ is defined to link them. $P_{2, k}$ is also duplicated into $s_{1}, P_{2, k}^{s_{1}}$, and $w_{2, k}$ connects them.

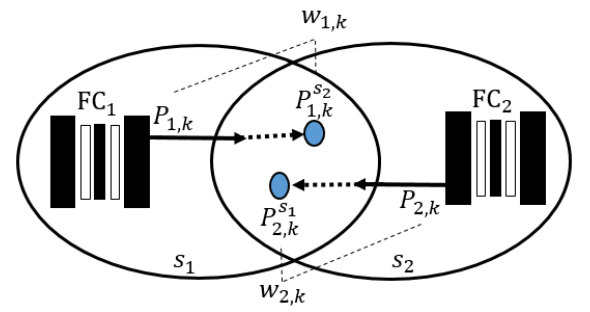

Fig. 4. The description of real, virtual, and global variables in the MFCS. Equations (10.a) and (10.b) guarantee that the duplicated powers in $s_{1}$ and $s_{2}$ are equal with each other [44].

$$
\begin{aligned}
& P_{1, k}-w_{1, k}=0, P_{2, k}^{s_{1}}-w_{2, k}=0, \\
& P_{1, k}^{s_{2}}-w_{1, k}=0, P_{2, k}-w_{2, k}=0,
\end{aligned}
$$

After defining the global power variable constraints, the distributed parallel form of C-ADMM is defined by (11) and (12).

The $F C_{1}$ module:

$$
\begin{gathered}
P_{1, k}^{j+1}, P_{2, k}^{s_{1}, j+1}=\min \left(c_{1, k}\left(P_{1, k}, P_{2, k}^{s_{1}}\right)+c_{B, k}\left(P_{B, k}\right)+\right. \\
\lambda_{1}^{s_{1}, j} P_{1, k}+\lambda_{2}^{s_{1}, j} P_{2, k}^{s_{1}}+ \\
\left.\frac{\rho}{2}\left(\left(P_{1, k}-w_{1, k}^{j}\right)^{2}+\left(P_{2, k}^{s_{1}}-w_{2, k}^{j}\right)^{2}\right)\right), \\
w_{1, k}^{j+1}=\frac{P_{1, k}^{j+1}+P_{1, k}^{s_{2}, j+1}}{2}, \\
\lambda_{1, k}^{s_{1}, j+1}=\lambda_{1, k}^{s_{1}, j}+\rho\left(P_{1, k}^{j+1}-w_{1, k}^{j+1}\right), \\
\lambda_{2, k}^{s_{1}, j+1}=\lambda_{2, k}^{s_{1, j}}+\rho\left(P_{2, k}^{j+1}-w_{2, k}^{j+1}\right), \\
\text { The } F C_{2} \text { module: } \\
P_{1, k}^{s_{2}, j+1}, P_{2, k}^{j+1}=m_{i n}\left(c_{2, k}\left(P_{1, k}^{s_{2}}, P_{2, k}\right)+c_{B, k}\left(P_{B, k}\right)+\right. \\
\lambda_{1}^{s_{2}, j} P_{1, k}^{s_{2}}+\lambda_{2}^{s_{2}, j} P_{2, k}+ \\
\left.\frac{\rho}{2}\left(\left(P_{1, k}^{s_{2}}-w_{1, k}^{j}\right)^{2}+\left(P_{2, k}-w_{2, k}^{j}\right)^{2}\right)\right), \\
w_{2, k}^{j+1}=\frac{P_{2, k}^{j+1}+P_{2, k}^{s_{1}, j+1}}{2} \\
\lambda_{1, k}^{s_{2}, j+1}=\lambda_{1, k}^{s_{2, j}}+\rho\left(P_{1, k}^{j+1}-w_{1, k}^{j+1}\right), \\
\lambda_{2, k}^{s_{2}, j+1}=\lambda_{2, k}^{s_{2, j}}+\rho\left(P_{2, k}^{j+1}-w_{2, k}^{j+1}\right),
\end{gathered}
$$

where $\lambda_{m, k}$ represents the Lagrangian multipliers, and $\rho$ is a positive tuning value. Equations (11.b) and (12.b) define the global power vector, $w_{G, k}$, calculated based on the average of all the linked modules, and $j$ denotes the number of iterations [44]. The optimization convergence is defined by (13), where $\mu_{1}$ and $\mu_{2}$ are the limiting values.

$$
\left\|\lambda_{m, k}^{j+1}-\lambda_{m, k}^{j}\right\|_{2}^{2} \leq \mu_{1}, \rho\left\|P_{m, k}^{j+1}-P_{m, k}^{j}\right\|_{2}^{2} \leq \mu_{2}
$$

Fig. 5 presents the flowchart of the C-ADMM algorithm in four steps.

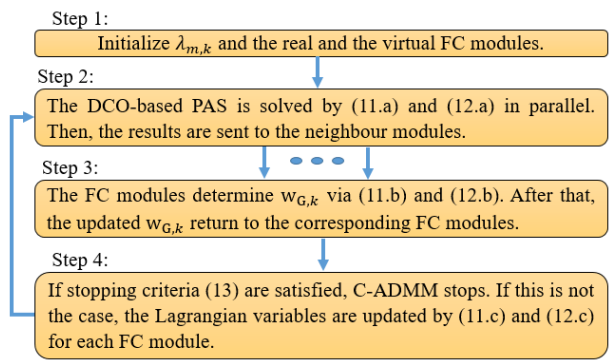

Fig. 5. The step-by-step flowchart of the decentralized C-ADMM algorithm. 
Fig.6 depicts the flow of information in C-ADMM, where the virtual powers are sent to the neighboring modules. Then, the global powers are calculated and returned in parallel.
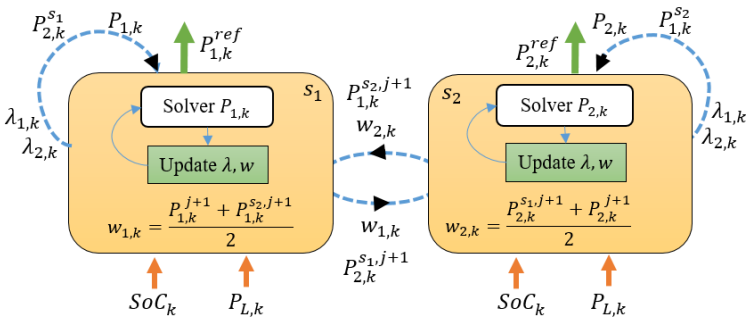

Fig. 6. The information flow between the FC modules via C-ADMM.

\section{B. PJ-ADMM-based PAS}

To improve the convergence of ADMM, PJ-ADMM is proposed in [45]. The formulation and parallel updating procedure are presented as:

The $F C_{1}$ module:

$$
\begin{gathered}
P_{1, k}^{j+1}, P_{2, k}^{s_{1}, j+1}=\min \left(\mathrm{c}_{1, k}\left(P_{1, k}, P_{2, k}^{S_{1}}\right)+\mathrm{c}_{B, k}\left(P_{B, k}\right)+\right. \\
\frac{\rho}{2}\left\|P_{1, k}-P_{1, k}^{S_{2}, j}-\lambda_{1, k}^{j}\right\|_{2}^{2}+\frac{1}{2}\left\|P_{1, k}-P_{1, k}^{j}\right\|_{P x i}^{2}+ \\
\left.\frac{\rho}{2}\left\|P_{2, k}^{s_{1}}-P_{2, k}^{j}-\lambda_{2, k}^{j}\right\|_{2}^{2}+\frac{1}{2}\left\|P_{2, k}^{S_{1}}-P_{2, k}^{S_{1}, j}\right\|_{P x i}^{2}\right), \\
\lambda_{1, k}^{j+1}=\lambda_{1, k}^{j}-\gamma\left(P_{1, k}^{j+1}-P_{1, k}^{s_{2}, j+1}\right), \\
\lambda_{2, k}^{j+1}=\lambda_{2, k}^{j}-\gamma\left(P_{2, k}^{S_{1}, j+1}-P_{2, k}^{j+1}\right),
\end{gathered}
$$

The $F C_{2}$ module:

$$
\begin{gathered}
P_{1, k}^{S_{2}, j+1}, P_{2, k}^{j+1}=\min \left(\mathrm{c}_{2, k}\left(P_{1, k}^{S_{2}}, P_{2, k}\right)+\mathrm{c}_{B, k}\left(P_{B, k}\right)+\right. \\
\frac{\rho}{2}\left\|P_{1, k}^{S_{2}}-P_{1, k}^{j}-\lambda_{1, k}^{j}\right\|_{2}^{2}+\frac{1}{2}\left\|P_{1, k}^{S_{2}}-P_{1, k}^{s_{2}, j}\right\|_{P x i}^{2}{ }^{+} \\
\left.\frac{\rho}{2}\left\|P_{2, k}-P_{2, k}^{s_{1}, j}-\lambda_{2, k}^{j}\right\|_{2}^{2}+\frac{1}{2}\left\|P_{2, k}-P_{2, k}^{j}\right\|_{P x i}^{2}\right), \\
\lambda_{1, k}^{j+1}=\lambda_{1, k}^{j}-\gamma\left(P_{1, k}^{s_{2}, j+1}-P_{1, k}^{j+1}\right), \\
\lambda_{2, k}^{j+1}=\lambda_{2, k}^{j}-\gamma\left(P_{2, k}^{j+1}-P_{2, k}^{S_{1}, j+1}\right),
\end{gathered}
$$

where Pxi presents a positive and symmetric semi-definite matrix, and $\gamma$ indicates a positive damping parameter. Fig. 7 and Fig. 8 illustrate the step-by-step update process and the inter-module communications of the DCO-based PAS.

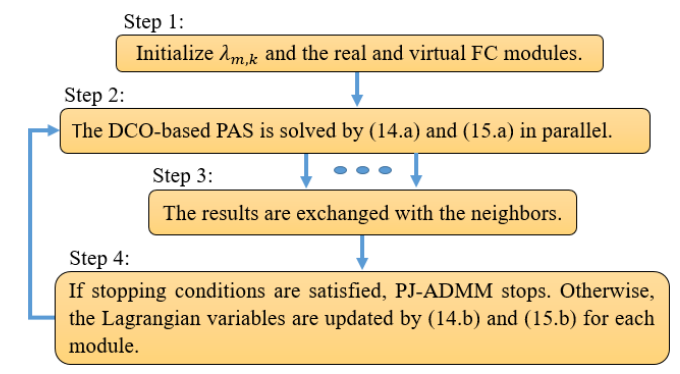

Fig. 7. The update process of the PJ-ADMM-based PAS.

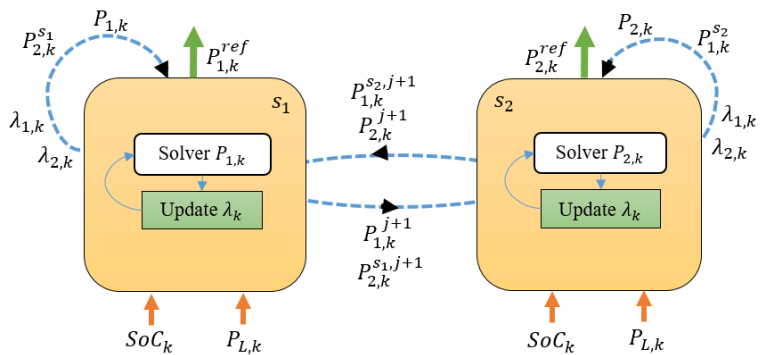

Fig. 8. The parallel communications of the decentralized PJ-ADMM PAS.

\section{SIMULATION RESULTS AND DISCUSSION}

To unveil the capability of the DCO algorithms, firstly, a comprehensive analysis of the two proposed PASs is performed in MATLAB software. In this sense, the developed approaches are compared with DP as an offline optimization method and SQP as a centralized convex programming algorithm. Subsequently, due to the inherent sensitivity of the ADMMbased optimization approaches to parameter tuning, this critical point is investigated for PJ-ADMM and C-ADMM. Next, the most potential algorithm is selected to scrutinize the effect of price sensitivity and fault occurrence in the performance of the PAS. It is essential to mention that the computational time extensively depends on the utilized PC hardware (Processor= Corei5, 2.30GHz, RAM=4.00GB). Except for the parameter tuning analysis subsection, the same initial values are applied to all the considered cases to establish an unbiased comparison.

\section{A. Optimal performance analysis}

A real driving cycle is utilized to inspect the performance of the developed DCO-based PASs, as shown in Fig. 9. Since the maximum power of the selected driving profile is higher than the developed test bench limitations, the power profile is scaled down by ten during the simulation and implementation steps.
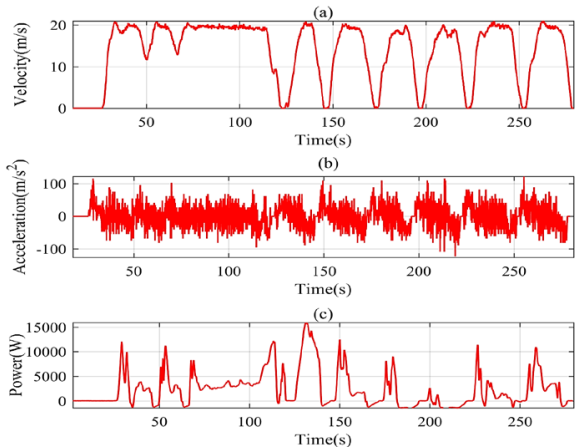

Fig. 9. The real driving profile: (a) velocity, (b) acceleration, and (c) power.

The optimized power trajectories using C-ADMM and PJADMM are shown in Fig. 10(a) and Fig. 10(b). As it can be seen, the modules collaborate and primarily operate at the efficient regions to fulfill the requested power and minimize the multi-objective cost functions. However, due to the slow response of the modules, the secondary source supplies the fast dynamic response and peaks. Fig. 10(c) compares the $S o C$ variations obtained by C-ADMM and PJ-ADMM. The $S O C$ of the battery pack oscillates between $69 \%$ and $72 \%$, less than approximately $3 \%$ variation. Although the obtained SoC variations by $\mathrm{C}-\mathrm{ADMM}$ and $\mathrm{PJ}-\mathrm{ADMM}$ are similar, a slight deviation can be observed in the $S o C$ levels from $50 \mathrm{~s}$ to $150 \mathrm{~s}$. It is due to more cumulative costs in the PJ-ADMM algorithm. After $150 \mathrm{~s}$, the $S o C$ fluctuations become almost equal because the responses of the DCO-based algorithms are similar. Another point is that the drawn power from the $\mathrm{FC}$ modules are increased after $250 \mathrm{~s}$ to keep the $S o C$ fluctuations close enough to the initial values. 

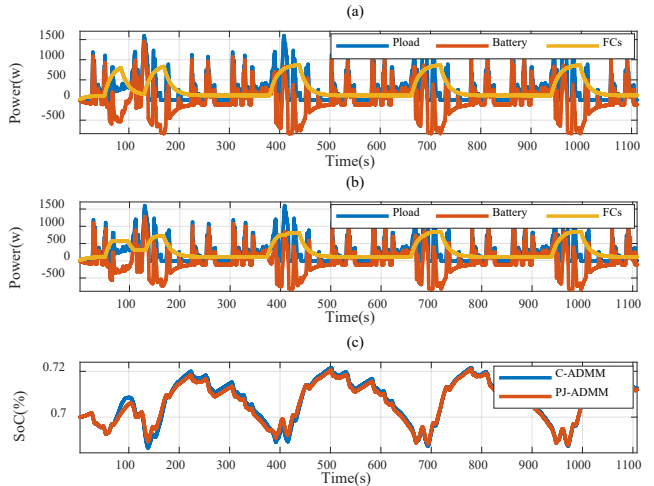

Fig. 10. Optimal PASs results (Pload: the requested power, $F C s$ : the power provided by the modules, Battery: the battery power) : (a) the profiles based on C-ADMM, (b) the profiles based on PJ-ADMM, and (c) the $S o C$ evaluations.

As an example, the time series and the distribution of the power in the real and virtual FCs are presented for C-ADMM case study in Fig. 11. $P_{1}$ and $P_{1}^{S_{2}}$ are the real and the virtual power profile of $F C_{1}$, and $P_{2}$ and $P_{2}^{s_{1}}$ are the real and the virtual power profile of $F C_{2}$, respectively. It is evident from this figure that the real power and virtual power of the modules are wellmatched. Moreover, the request power from the FCs is almost located in high efficient regions. Since the convergence speed of the C-ADMM algorithm is faster than the modular powertrain dynamics, the virtual power of FCs gets very close to the real one. It can also be realized that since $F C_{2}$ has a higher level of maximum output power and efficiency, it is more utilized than $F C_{1}$.
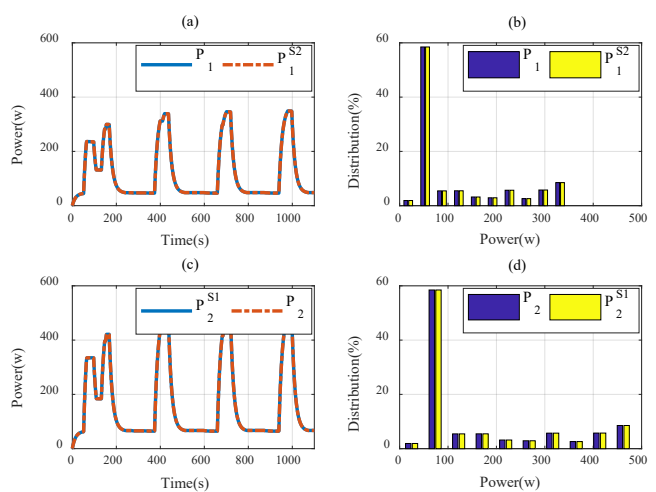

Fig. 11. The optimized power and power distributions of the modules using C-ADMM: (a) the power profile of $F C_{1}$, (b) the distribution of $F C_{1}$, (c) the power profile of $\mathrm{FC}_{2}$, and (d) the distribution of $\mathrm{FC}_{2}$.

The computation time and the number of iterations according to $F C_{1}$ are illustrated in Fig.12.
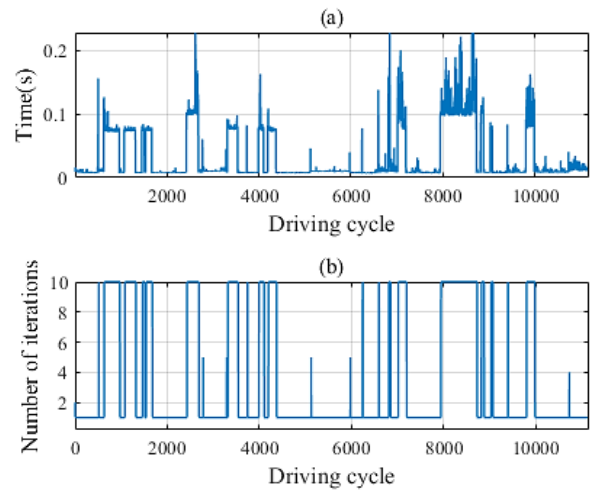

Fig. 12. (a) The computational time, and (b) the number of iterations.
The detailed computing performance and final price of the developed PASs are presented in Table II, where $\mathrm{T}$ is the computation time per second, and \# is the number of iterations. As shown in Table II, the proposed DCO algorithms are marginally better than SQP and have shown a very close performance to DP in terms of the total cost. However, in terms of computational time, the decentralized structures of the proposed PASs have made them much faster than SQP, where the computational burdens have been reduced by $78.4 \%$ and $84.1 \%$ concerning C-ADMM and PJ-ADMM, respectively. The computational time in PJ-ADMM is 5.7\% lower than CADMM because of the proximal term [45]. SQP is the slowest optimization method with an operation time per iteration of 0.08 s. C-ADMM and PJ-ADMM are faster than SQP with 0.04 $\mathrm{s}$ and $0.03 \mathrm{~s}$, respectively. The associated total costs based on PJ-ADMM and C-ADMM are $\$ 0.0426$ and $\$ 0.0432$, which are $4.4118 \%$ and $5.8824 \%$ higher than DP, respectively. These minor differences are derived from the single-step optimization and the convex modeling approximations. The hydrogen consumptions are the highest cost, where $F C_{1}$ and $F C_{2}$ contribute $\$ 0.0173$ and $\$ 0.0109$ under PJ-ADMM and $\$ 0.0174$ and $\$ 0.010$ under C-ADMM, respectively. The second-largest expenses are related to the modules' degradations, which are approximately $\$ 0.0096$ and $\$ 0.0098$ by PJ-ADMM and CADMM, respectively. The degradation costs of the battery pack using PJ-ADMM and C-ADMM are about $\$ 0.0048$ and $\$ 0.005$, respectively, which constitute $11.3 \%$ and $11.6 \%$ of the final costs. The C-ADMM and PJ-ADMM cost terms equally decline because of the normalized cost functions. It should be pronounced that DCOs can assist the modules to prolong the FCSs lifetime and minimize the final costs. Fig. 13 provides the optimized cumulative cost changes by utilizing the PJ-ADMM algorithm.

Table II

The detailed computing performance and final price

\begin{tabular}{ccccccc}
\hline \hline & DP & SQP & \multicolumn{2}{c}{ C-ADMM } & \multicolumn{2}{c}{ PJ-ADMM } \\
& $F C_{1}+F C_{2}$ & $F C_{1}+F C_{2}$ & $F C_{1}$ & $F C_{2}$ & $F C_{1}$ & $F C_{2}$ \\
\hline $\mathrm{T}$ & 4160.5 & 208.4 & 45.9 & 45.9 & 31.7 & 33.6 \\
\hline$\#$ & - & 2463 & 968 & 972 & 932 & 963 \\
\hline$S_{H, m}$ & 0.0254 & 0.0298 & 0.0174 & 0.01 & 0.0173 & 0.0109 \\
\hline $\mathrm{S}_{d, m}$ & 0.0091 & 0.0101 & 0.0062 & 0.0039 & 0.0062 & 0.0036 \\
\hline$S_{B}$ & 0.0055 & 0.0043 & 0.0051 & 0.0041 \\
\hline$S_{S o C}$ & 0 & 0.0008 & 0.0006 & 0.0005 \\
\hline$S_{T}$ & 0.040 & 0.045 & 0.0432 & 0.0426 \\
\hline \hline
\end{tabular}

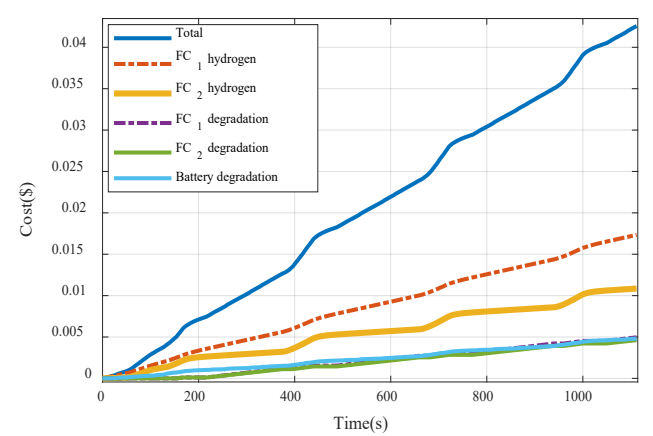

Fig. 13. The cost evaluation of the PJ-ADMM algorithm. 


\section{B. Parameter tuning analysis}

In this subsection, the effects of the DCO-based PASs parameters on the total cost and the computational time are cautiously examined. A set of simulations are performed to seek appropriate ranges for $\rho$ and $\mu$. The obtained outcomes are presented in Fig. 14. Overall, the costs present upward trends with some fluctuations when $\rho$ increases and $\mu$ declines. Furthermore, the computational time experiences incremental trends in both cases, specifically when $\mu$ passes $1.0 \times 10^{-6}$. Consequently, the selected $\rho$ and $\mu$ ranges require a balance between the final cost and the data processing efficiency.
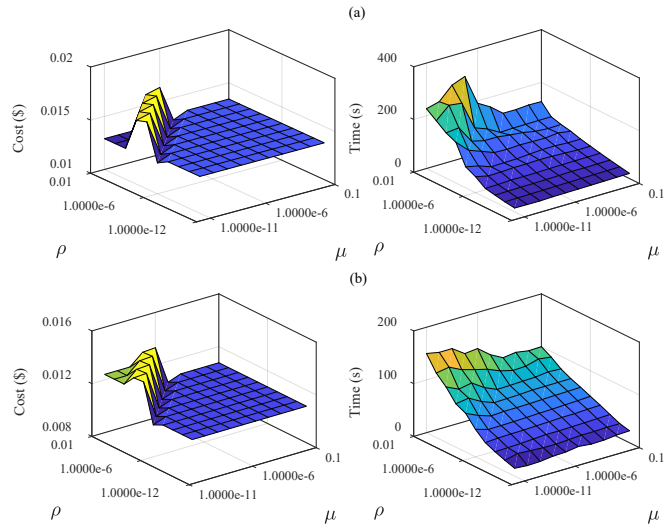

Fig. 14. Variation with $\rho$ and $\mu$ of the final cost and the processing time: (a) C-ADMM and (b) PJ-ADMM.

\section{Price sensitivity and optimization criteria analysis}

For the sake of exploring the influence of price changes and optimization goals over the behavior of the DCO-based PASs, a straightforward and effective investigation of PJ-ADMM is performed in this section. Fig. 15 illustrates the cost and optimization criteria evaluation. The current situation in 2020, shown by the red dashed line, is considered the baseline. The upcoming trend after five years in 2025 is considered a means of comparison with two different probable case studies. The first case study is related to a cheaper PEMFC stack price (-20 $\%)$ trend, which is plotted by the blue dashed line because of applying several cost-effective strategies from FC stack manufacturers. The second case study assumes a surge in the hydrogen price $(+20 \%)$, depicted by the yellow-solid line, due to the growth of the FCV production numbers and the penetration of the hydrogen-powered system life. The criteria of the DCO-based algorithms are defined through $\eta$, where $\eta=0$ shows the optimization is only hydrogenconsumption-oriented, and $\eta=1$ means the optimization is only health-aware-oriented. It is pronounced that compared to the baseline price, a $20 \%$ decrease in the FC stack price reduces the minimal value to around $7.32 \%$.

Moreover, a $20 \%$ increase in the hydrogen price augments the optimal value to around $9.76 \%$. The optimal $\eta=0.18$ is increased in both of the case studies. In future, adaptive and comprehensive DCO PASs can be established while considering short-term and long-term price trajectories.

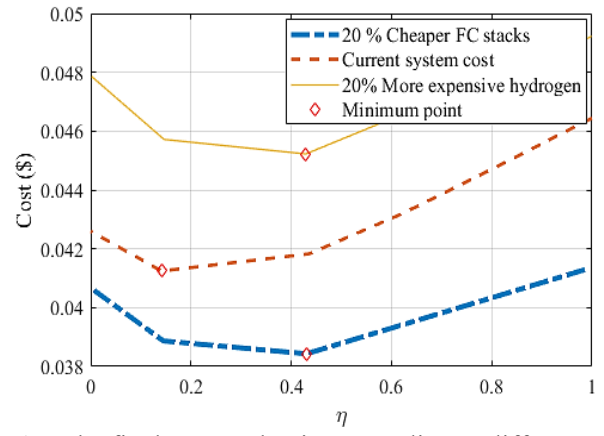

Fig. 15. The final cost evaluation according to different price and optimization objective scenarios.

\section{Fault-resilient analysis}

To evaluate the robustness and modularity (plug \& play), a comparison between regular and faulty operation of PJ-ADMM is performed in this subsection. This paper takes only electrical fault conditions into account, which will affect the system's total output power. For this purpose, a dynamic electrical fault is imposed to $F C_{2}$ for 100 s. Fig. 16 depicts the power trajectories and the $S o C$ fluctuations of the well-behaved and misbehaved modules during and after the electrical fault. Throughout the fault occurrence, the functional module and battery unit collaborate to converge to the optimal power. After passing the electrical fault condition, due to the parallel structure of PJ-ADMM, this algorithm can conveniently follow the requested power profile. Therefore, if one of the modules stops regular operation for a specific duration and gets back to regular operation again, the Dec-PAS can keep working without making any problems for the powertrain system. It is an intriguing clue that applying these decentralized approaches at module-level fault operation needs further investigations.

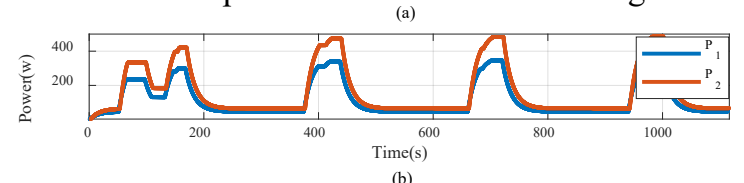

(b)

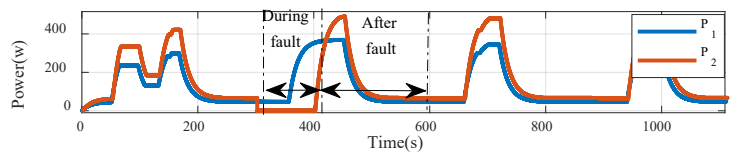

(C)

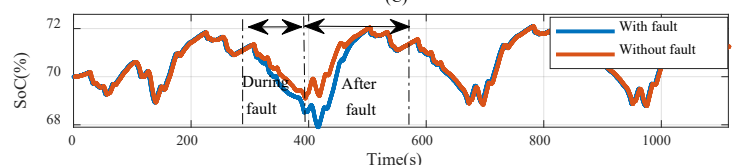

Fig. 16. A comparison between standard and fault operations of the Dec-PAS based on PJ-ADMM: (a) the power profile evolutions in case of regular operation, (b) the power profile trajectories in case of dynamic electrical fault operation, and (c) the $S o C$ levels of the battery pack.

\section{EXPERIMENTAL IMPLEMENTATION}

An experimental test based on FPGA implementation is conducted under the real driving profile to verify the simulation results. In this regard, PJ-ADMM is singled out due to its superior performance compared to C-ADMM. Generally, the computational time and performance in the simulation step running on general-purpose hardware (PC) differ from a realtime field test for several reasons, such as processing capacity, 
memory, communication delay, and uncertainty in the powertrain components. The power trajectories and $\mathrm{SoC}$ oscillation are demonstrated in Fig. 17. Moreover, the power distributions are shown in Fig. 18. The final cost of the PJADMM-based PAS shows an extra cost of $6.62 \%$ compared to that of the offline one. The hydrogen consumption cost approximately forms $41.15 \%$ of the final cost. The degradation of FC modules is the second-highest cost, with about $27.84 \%$ of the final cost. The modular system operates in the low-power and high-efficiency region to mitigate the degradation expense and reduce hydrogen consumption. The suggested PAS works well to ensure the constraints of the powertrain components prolong their lifespans. The computational complexity of PJADMM is about $63.25 \%$ lower than SQP. In this regard, it is a practical and suitable optimization algorithm for low-cost systems with limited onboard computational power. These results highlight the potential of the decentralized implementation schemes in real-time applications.
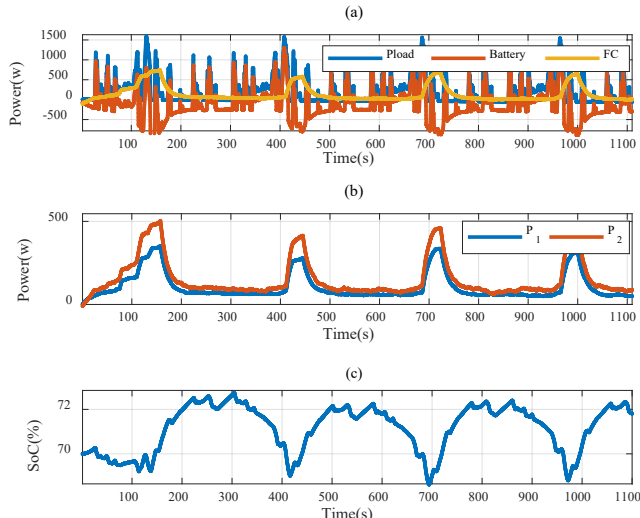

Fig. 17. The implementation results of the developed PAS based on PJADMM: (a) the power profiles, (b) the modules' power profiles, and (c) the $\mathrm{SoC}$ level fluctuations.
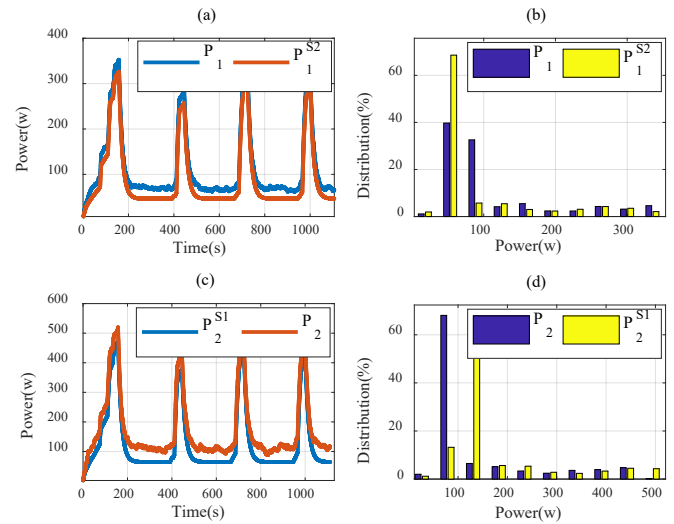

Fig. 18. The experimental results of the PJ-ADMM approach: (a) the power profile of $F C_{1}$, (b) the distribution of $F C_{1}$, (c) the power profile of $F C_{2}$, and (d) the distribution of $F C_{2}$.

\section{CONCLUSION}

This paper presents a comprehensive analysis of two DecPASs based on distributed convex optimization in an MFCV application. The two decomposition-based algorithms (CADMM and PJ-ADMM) are selected due to their parallel updating optimization procedures and their global convergences. In this regard, a general PAS optimization problem with a convex approximation is formulated for a modular powertrain system, including hydrogen consumption and lifetime of the FC modules as well as the lifetime of the battery unit. After that, the decentralized optimization frameworks for solving the power-splitting problem are presented. To evaluate the performance of the proposed DecPASs under real driving profile, an in-depth comparative analysis of costs and computational times are presented compared to DP and SQP.

Additionally, due to the importance of parameter tuning in the ADMM-based optimization algorithms, this feature in CADMM and PJ-ADMM is investigated. Since the PJ-ADMM algorithm has reached a better general performance than CADMM under the discussed scenarios, it is also used for two further sensitivity analyses in terms of dynamic fault and price fluctuation case studies. Finally, the experimental results unveil that the implemented PJ-ADMM decentralized scheme achieves excellent performance compared to SQP. Considering the outcomes of this manuscript, the following directions are put forward for future endeavors:

- The inclusion of future trip information in the DCObased PAS framework. This idea requires developing a predictive-based control strategy and introducing a trip model with a high level of reliability and accuracy.

- The integration of thermal models of FCSs and battery into the Dec-PAS and scrutinizing it from the perspective of different initial temperatures and health conditions.

- Combining DCO-based PASs with fault detection algorithms to develop robust strategies for MFCV powertrains.

\section{REFERENCES}

Z. P. Cano, D. Banham, S. Ye, A. Hintennach, J. Lu, M. Fowler, et al., "Batteries and fuel cells for emerging electric vehicle markets," Nature Energy, vol. 3, pp. 279-289, 2018/04/01 2018.

H. S. Das, C. W. Tan, and A. H. M. Yatim, "Fuel cell hybrid electric vehicles: A review on power conditioning units and topologies," Renewable and Sustainable Energy Reviews, vol. 76, pp. 268-291, 2017/09/01/ 2017.

T. Hua, R. Ahluwalia, L. Eudy, G. Singer, B. Jermer, N. AsselinMiller, et al., "Status of hydrogen fuel cell electric buses worldwide," Journal of Power Sources, vol. 269, pp. 975-993, 2014/12/10/ 2014.

U. Eberle, B. Müller, and R. Helmolt, "Fuel cell electric vehicles and hydrogen infrastructure: Status 2012," Energy \& Environmental Science, vol. 5, pp. 8790-8798, 07/30 2012.

X. Hu, C. Zou, C. Zhang, and Y. Li, "Technological Developments in Batteries: A Survey of Principal Roles, Types, and Management Needs," IEEE Power and Energy Magazine, vol. 15, pp. 20-31, 2017.

N. Sulaiman, M. A. Hannan, A. Mohamed, E. H. Majlan, and W. R. Wan Daud, "A review on energy management system for fuel cell hybrid electric vehicle: Issues and challenges," Renewable and Sustainable Energy Reviews, vol. 52, pp. 802-814, 2015/12/01/ 2015.

H. Ramírez-Murillo, C. Restrepo, J. Calvente, A. Romero, and R. Giral, "Energy Management of a Fuel-Cell Serial-Parallel Hybrid System," IEEE Transactions on Industrial Electronics, vol. 62, pp. 5227-5235, 2015.

M. G. Carignano, V. Roda, R. Costa-Castelló, L. Valino, A. Lozano, and F. Barreras, "Assessment of energy management in a fuel 
cell/battery hybrid vehicle," ieee access, vol. 7, pp. 16110-16122, $1 / 1 / 20192019$.

C. Wu, J. Chen, C. Xu, and Z. Liu, "Real-Time Adaptive Control of a Fuel Cell/Battery Hybrid Power System With Guaranteed Stability," IEEE Transactions on Control Systems Technology, vol. 25, pp. 1394-1405, 2017.

[10] S. Ziaeinejad, Y. Sangsefidi, and A. Mehrizi-Sani, "Fuel Cell-Based Auxiliary Power Unit: EMS, Sizing, and Current Estimator-Based Controller," IEEE Transactions on Vehicular Technology, vol. 65, pp. 4826-4835, 2016.

[11] X. Meng, Q. Li, G. Zhang, T. Wang, W. Chen, and T. Cao, "A DualMode Energy Management Strategy Considering Fuel Cell Degradation for Energy Consumption and Fuel Cell Efficiency Comprehensive Optimization of Hybrid Vehicle," IEEE Access, vol. 7, pp. 134475-134487, 2019.

[12] R. Wai, S. Jhung, J. Liaw, and Y. Chang, "Intelligent Optimal Energy Management System for Hybrid Power Sources Including Fuel Cell and Battery," IEEE Transactions on Power Electronics, vol. 28, pp. 3231-3244, 2013.

[13] M. Kandidayeni, A. O. M. Fernandez, A. Khalatbarisoltani, L. Boulon, S. Kelouwani, and H. Chaoui, "An Online Energy Management Strategy for a Fuel Cell/Battery Vehicle Considering the Driving Pattern and Performance Drift Impacts," IEEE Transactions on Vehicular Technology, vol. 68, pp. 11427-11438, 2019.

[14] M. Kandidayeni, A. M. Fernandez, L. Boulon, and S. Kelouwani, "Efficiency Upgrade of Hybrid Fuel Cell Vehicles' Energy Management Strategies by Online Systemic Management of Fuel Cell," IEEE Transactions on Industrial Electronics, pp. 1-1, 2020.

[15] H. Chen, J. Chen, Z. Liu, and H. Lu, "Real-time optimal energy management for a fuel cell/battery hybrid system," asian journal of control, vol. 21, pp. 1847-1856, 7/1/2019 2019.

[16] Y. Yan, Q. Li, W. Chen, B. Su, J. Liu, and L. Ma, "Optimal Energy Management and Control in Multimode Equivalent Energy Consumption of Fuel Cell/Supercapacitor of Hybrid Electric Tram," IEEE Transactions on Industrial Electronics, vol. 66, pp. 60656076, 2019.

[17] X. Han, H. He, J. Wu, J. Peng, and Y. Li, "Energy management based on reinforcement learning with double deep Q-learning for a hybrid electric tracked vehicle," Applied Energy, vol. 254, p. 113708, 2019.

[18] E. Tazelaar, B. Veenhuizen, P. vandenBosch, and M. Grimminck, "Analytical Solution of the Energy Management for Fuel Cell Hybrid Propulsion Systems," IEEE Transactions on Vehicular Technology, vol. 61, pp. 1986-1998, 2012.

[19] X. Hu, N. Murgovski, L. M. Johannesson, and B. Egardt, "Optimal dimensioning and power management of a fuel cell/battery hybrid bus via convex programming," IEEE/ASME transactions on mechatronics, vol. 20, pp. 457-468, 2014.

[20] D. Zhou, A. Al-Durra, F. Gao, A. Ravey, I. Matraji, and M. Godoy Simões, "Online energy management strategy of fuel cell hybrid electric vehicles based on data fusion approach," Journal of Power Sources, vol. 366, pp. 278-291, 2017/10/31/ 2017.

[21] X. Hu, C. Zou, X. Tang, T. Liu, and L. Hu, "Cost-optimal energy management of hybrid electric vehicles using fuel cell/battery health-aware predictive control," ieee transactions on power electronics, pp. 1-1, 1/1/2019 2019.

[22] B. Geng, J. K. Mills, and D. Sun, "Two-Stage Energy Management Control of Fuel Cell Plug-In Hybrid Electric Vehicles Considering Fuel Cell Longevity," IEEE Transactions on Vehicular Technology, vol. 61, pp. 498-508, 2012.

[23] H. Chen, J. Chen, H. Lu, C. Yan, and Z. Liu, "A Modified MPCbased Optimal Strategy of Power Management for Fuel Cell Hybrid Vehicles," IEEE/ASME Transactions on Mechatronics, 2020.

[24] J. Chen, C. Xu, C. Wu, and W. Xu, "Adaptive Fuzzy Logic Control of Fuel-Cell-Battery Hybrid Systems for Electric Vehicles," ieee transactions on industrial informatics, vol. 14, pp. 292-300, $1 / 1 / 20182018$

[25] A. K. Soltani, M. Kandidayeni, L. Boulon, and D. L. St-Pierre, "Modular Energy Systems in Vehicular Applications," Energy Procedia, vol. 162, pp. 14-23, 2019/04/01/ 2019.

[26] N. Marx, L. Boulon, F. Gustin, D. Hissel, and K. Agbossou, "A review of multi-stack and modular fuel cell systems: Interests, application areas and on-going research activities," International Journal of Hydrogen Energy, vol. 39, pp. 12101-12111, 2014.
J. E. Garcia, D. F. Herrera, L. Boulon, P. Sicard, and A. Hernandez, "Power sharing for efficiency optimisation into a multi fuel cell system," in 2014 IEEE 23rd International Symposium on Industrial Electronics (ISIE), 2014, pp. 218-223.

[28] N. Marx, D. Hissel, F. Gustin, L. Boulon, and K. Agbossou, "On the sizing and energy management of an hybrid multistack fuel cell Battery system for automotive applications," International Journal of Hydrogen Energy, vol. 42, pp. 1518-1526, 2017/01/12/ 2017.

[29] B. Somaiah and V. Agarwal, "Distributed Maximum Power Extraction From Fuel Cell Stack Arrays Using Dedicated Power Converters in Series and Parallel Configuration," IEEE Transactions on Energy Conversion, vol. 31, pp. 1442-1451, 2016.

[30] H. Zhang, X. Li, X. Liu, and J. Yan, "Enhancing fuel cell durability for fuel cell plug-in hybrid electric vehicles through strategic power management," Applied Energy, vol. 241, pp. 483-490, 2019/05/01/ 2019.

[31] A. O. M. Fernandez, M. Kandidayeni, L. Boulon, and H. Chaoui, "An Adaptive State Machine Based Energy Management Strategy for a Multi-Stack Fuel Cell Hybrid Electric Vehicle," IEEE Transactions on Vehicular Technology, pp. 1-1, 2019.

[32] T. Wang, Q. Li, H. Yang, L. Yin, X. Wang, Y. Qiu, et al., "Adaptive current distribution method for parallel-connected PEMFC generation system considering performance consistency," Energy Conversion and Management, vol. 196, pp. 866-877, 2019/09/15/ 2019.

[33] H. Yin, C. Zhao, and C. Ma, "Decentralized Real-Time Energy Management for a Reconfigurable Multiple-Source Energy System," ieee transactions on industrial informatics, vol. 14, pp. 4128-4137, 9/1/2018 2018.

[34] H. Yin, C. Zhao, M. Li, C. Ma, and M.-Y. Chow, "A Game Theory Approach to Energy Management of An EngineGenerator/Battery/Ultracapacitor Hybrid Energy System," ieee transactions on industrial electronics, vol. 63, pp. 4266-4277, 7/1/2016 2016.

[35] Q. Li, T. Wang, C. Dai, W. Chen, and L. Ma, "Power Management Strategy Based on Adaptive Droop Control for a Fuel Cell-BatterySupercapacitor Hybrid Tramway," IEEE Transactions on Vehicular Technology, vol. 67, pp. 5658-5670, 2018.

[36] D. K. Molzahn, F. Dörfler, H. Sandberg, S. H. Low, S. Chakrabarti, R. Baldick, et al., "A Survey of Distributed Optimization and Control Algorithms for Electric Power Systems," IEEE Transactions on Smart Grid, vol. 8, pp. 2941-2962, 2017.

[37] A. Kargarian, J. Mohammadi, J. Guo, S. Chakrabarti, M. Barati, G. Hug, et al., "Toward Distributed/Decentralized DC Optimal Power Flow Implementation in Future Electric Power Systems," IEEE Transactions on Smart Grid, vol. 9, pp. 2574-2594, 2018.

[38] D. P. Bertsekas and A. Scientific, Convex optimization algorithms: Athena Scientific Belmont, 2015.

[39] A. J. Conejo and J. A. Aguado, "Multi-area coordinated decentralized DC optimal power flow," IEEE transactions on power systems, vol. 13, pp. 1272-1278, 1998.

[40] G. Cohen, "Auxiliary problem principle and decomposition of optimization problems," Journal of optimization Theory and Applications, vol. 32, pp. 277-305, 1980.

[41] Z. Zhang and M.-Y. Chow, "Convergence analysis of the incremental cost consensus algorithm under different communication network topologies in a smart grid," IEEE Transactions on Power Systems, vol. 27, pp. 1761-1768, 2012.

[42] F. J. Nogales, F. J. Prieto, and A. J. Conejo, "A decomposition methodology applied to the multi-area optimal power flow problem," Annals of operations research, vol. 120, pp. 99-116, 2003.

[43] D. Gabay and B. Mercier, "A dual algorithm for the solution of nonlinear variational problems via finite element approximation," Computers \& mathematics with applications, vol. 2, pp. 17-40, 1976.

[44] S. Boyd, N. Parikh, E. Chu, B. Peleato, and J. Eckstein, "Distributed optimization and statistical learning via the alternating direction method of multipliers," Foundations and Trends ${ }^{\circledR}$ in Machine learning, vol. 3, pp. 1-122, 2011.

[45] W. Deng, M.-J. Lai, Z. Peng, and W. Yin, "Parallel multi-block ADMM with O (1/k) convergence," Journal of Scientific Computing, vol. 71, pp. 712-736, 2017. 
L. Liu and Z. Han, "Multi-block ADMM for big data optimization in smart grid," in 2015 International Conference on Computing, Networking and Communications (ICNC), 2015, pp. 556-561.

[47] S. East and M. Cannon, "Fast Optimal Energy Management With Engine On/Off Decisions for Plug-in Hybrid Electric Vehicles," presented at the IEEE Control Systems Letters, 2019.

[48] S. East and M. Cannon, "Energy Management in Plug-In Hybrid Electric Vehicles: Convex Optimization Algorithms for Model Predictive Control," ieee transactions on control systems and technology, pp. 1-13, 1/1/2019 2019.

[49] A. Khalatbarisoltani, M. Kandidayeni, L. Boulon, and X. Hu, "Power Allocation Strategy based on Decentralized Convex Optimization in Modular Fuel Cell Systems for Vehicular Applications," IEEE Transactions on Vehicular Technology, pp. 1$1,2020$.

[50] F. Martel, S. Kelouwani, Y. Dubé, and K. Agbossou, "Optimal economy-based battery degradation management dynamics for fuelcell plug-in hybrid electric vehicles," Journal of Power Sources, vol. 274, pp. 367-381, 2015/01/15/2015

[51] H. Chen, P. Pei, and M. Song, "Lifetime prediction and the economic lifetime of Proton Exchange Membrane fuel cells," Applied Energy, vol. 142, pp. 154-163, 2015/03/15/ 2015.

[52] N. Herr, J.-M. Nicod, C. Varnier, L. Jardin, A. Sorrentino, D. Hissel, et al., "Decision process to manage useful life of multi-stacks fuel cell systems under service constraint," Renewable Energy, vol. 105, pp. 590-600, 2017/05/01/ 2017.

[53] S. Satyapal, "U.S. Department of Energy Hydrogen and Fuel Cell Technology Overview " 2018.

[54] K. Mongird, V. V. Viswanathan, P. J. Balducci, M. J. E. Alam, V. Fotedar, V. S. Koritarov, et al., "Energy Storage Technology and Cost Characterization Report," Pacific Northwest National Lab.(PNNL), Richland, WA (United States)2019.

[55] USDRIVE, "Fuel Cell Technical Team Roadmap," 2017.
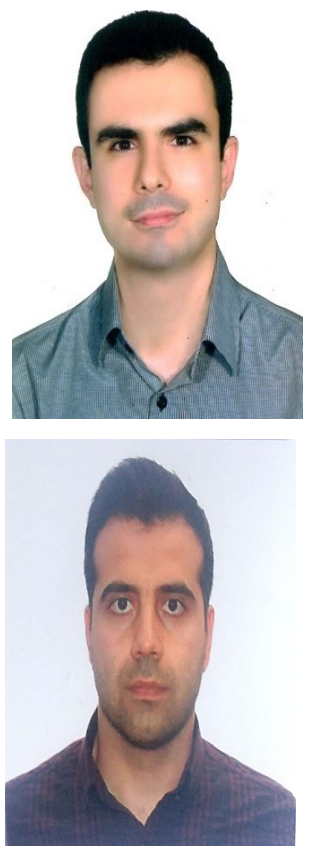

Arash Khalatbarisoltani (S'18) received the master's degree in mechatronics from Arak University, Arak, Iran, in 2016. He is currently working toward the Ph.D. degree with the Hydrogen Research Institute (IRH) and the Department of Electrical and Computer Engineering, Université du Québec à TroisRivières (UQTR), Trois-Rivières, QC, Canada. His research interests include optimal control, decentralized systems, fuel cell systems, energy management, renewable energy, and intelligent transport systems.

Mohsen Kandidayeni (S'18) was born in Tehran (Iran) in 1989. His educational journey has spanned through different paths. He received the B.S. degree in Mechanical Engineering in 2011 and then did a master's degree in Mechatronics at Arak University (Iran) in 2014. He joined the Hydrogen Research Institute of University of Quebec, Trois-Rivières (UQTR), QC, Canada, in 2016 and received his Ph.D. degree in Electrical Engineering from this university in 2020 . He is currently a postdoctoral researcher in ElectricTransport, Energy Storage, and Conversion Lab (e-TESC) at Université de Sherbrooke and a research assistant member in the Hydrogen Research Institute of UQTR. He has been the recipient of several awards/honors during his educational path, such as a doctoral scholarship from the Fonds de recherche du Québec-Nature et technologies (FRQNT), a postdoctoral scholarship from FRQNT, an excellence student grant from UQTR, and the $3^{\text {rd }}$ prize in Energy Research Challenge from the Quebec Ministry of Energy and Natural Resources. He has been actively involved in conducting research through authoring, coauthoring, and reviewing several papers in different prestigious scientific journals and also participating in various international conferences. His research interests include energy-related topics, such as hybrid electric vehicles, fuel cell systems, energy management, Multiphysics systems, modeling, and control.

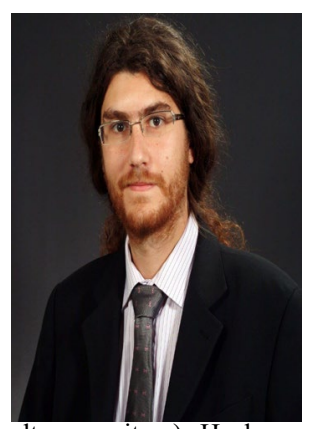

Loïc Boulon (M'10, SM'15) received the master's degree in electrical and automatic control engineering from the University of Lille (France) in 2006. Then, he obtained a Ph.D. in electrical engineering from the University of FrancheComté (France). Since 2010, he has been a professor at UQTR (Full Professor since 2016) and works in the Hydrogen Research Institute (Deputy director since 2019). His work deals with modeling, control, and energy management of multiphysics systems. His research interests include hybrid electric vehicles, energy and power sources (fuel cell systems, batteries, and ultracapacitors). He has published more than 120 scientific papers in peerreviewed international journals and international conferences and has given over 35 invited conferences all over the world. Since 2019, he is the world's most-cited author of the topic "Proton exchange membrane fuel cells (PEMFC); Fuel cells; Cell stack" in Elsevier SciVal. In 2015, Loïc Boulon was general chair of the IEEE-Vehicular Power and Propulsion Conference in Montréal (QC, Canada). Prof. Loïc Boulon is now VP-Motor Vehicles of the IEEE Vehicular Technology Society, and he found the "International Summer School on Energetic Efficiency of Connected Vehicles" and the "IEEE VTS Motor Vehicle Challenge". He is the holder of the Canada Research Chair in Energy Sources for the Vehicles of the future.

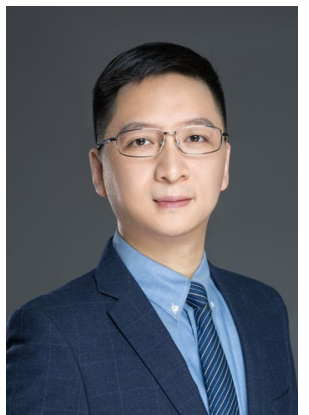

Xiaosong Hu (SM'16) received the Ph.D. degree in automotive engineering from the Beijing Institute of Technology, Beijing, China, in 2012. He did scientific research and completed the Ph.D. dissertation in Automotive Research Center at the University of Michigan, Ann Arbor, MI, USA, between 2010 and 2012 .

He is currently a Professor with the Department of Mechanical and Vehicle Engineering, Chongqing University, Chongqing, China. He was a Postdoctoral Researcher with the Department of Civil and Environmental Engineering, University of California, Berkeley, CA, USA, between 2014 and 2015, as well as at the Swedish Hybrid Vehicle Center and the Department of Signals and Systems at Chalmers University of Technology, Gothenburg, Sweden, between 2012 and 2014. He was also a Visiting Postdoctoral Researcher with the Institute for Dynamic Systems and Control at Swiss Federal Institute of Technology (ETH), Zurich, Switzerland, in 2014. His research interests include modeling and control of alternative powertrains and energy storage systems.

Dr. Hu has been the recipient of numerous prestigious awards/honors, including Web of Science Highly-Cited Researcher by Clarivate Analytics, SAE Environmental Excellence in Transportation Award, IEEE ITSS Young Researcher Award, SAE Ralph Teetor Educational Award, Emerging Sustainability Leaders Award, EU Marie Currie Fellowship, ASME DSCD Energy Systems Best Paper Award, and Beijing Best Ph.D. Dissertation Award. He is an IET Fellow. 